\title{
An Introduction to Health Systems
}

DAVID MCCOY AND PASCALE ALLOTEY

\subsection{Introduction}

Health systems are complex. For one, they are made up of multiple inter-acting components. Indeed, according to the World Health Organization (WHO), a health system consists of all 'organizations, people and actions whose primary intent is to promote, restore or maintain health'. They are also usually defined as country-level entities, rendering them large, encompassing both rural and urban areas, public and private systems as well as formal/allopathic and informal/traditional systems of health provision.

Health systems perform multiple functions in society - they do not merely deliver healthcare services and other interventions aimed at maintaining or improving health. They play a role in protecting households from the financial impacts of both illness and the costs of healthcare. It is important to note that health systems also perform an economic function in society (Sachs, 2001). For example, there is some evidence that the health of a population can influence economic productivity, while for many health workers and businesses, the health system is an economic sector that provides employment, wages and business opportunities. Health systems are also social and cultural institutions that play a function in helping establish 'a wider set of societal norms and values' (Gilson, 2003).

Health systems are open systems that exist in a dynamic relationship with their wider context. Indeed, they are diffuse systems with poorly defined and often porous boundaries and are thus adaptive and continuously evolving in response to multiple factors. For this reason, the social, political and economic context of any health system has to be considered when assessing its structure and performance.

Finally, health systems are sites of competition and contestation between actors with different needs and wants. There is contestation 
over how health priorities are set, how health systems are financed and how resources are allocated within the system. There are often ideologically and politically contrasting visions of what role a health system should play in society and what role the state and the market should play within health systems.

These different aspects of the complexity of health systems are rarely addressed simultaneously and in an inter-disciplinary manner. The truth is that health systems can only be comprehensively studied and understood through multiple disciplinary lenses, including those of history, economics, medicine, epidemiology, politics, law, ethics, anthropology and sociology.

This book presents a comprehensive and critical analysis of health systems within the context of local politics, history and socioeconomic development. In the following sub-sections, we unpack some of the different dimensions of health systems complexity. First, we look at the different societal functions performed by the health system. We then investigate how health systems are also sites of contestation between different ideas and values as well as different interest groups. We then examine a variety of approaches to constructing health systems frameworks and typologies and how these can be used to describe and understand the functioning and performance of health systems. The next section discusses the open and contextual nature of health systems and the relationship between the health system and a variety of external factors, including shifts in international health policy-making. The chapter concludes with a brief discussion of systems thinking, which is discussed in greater detail in Chapter 2.

\subsection{The Different Societal Functions of a Health System}

For most, the obvious function of a health system is to deliver a variety of services and interventions. These services may be 'personal' services (delivered to individuals or families) or 'non-personal' services (typically public health interventions targeted at entire populations or the environment in which people live). Clearly, this is a primary function of health systems, and most evaluations of health systems performance are based on how well these personal and non-personal services are delivered in terms of their effectiveness, accessibility, fairness, efficiency and affordability. 
However, health systems are more than just a vehicle for delivering health services. For example, health systems can help define and shape the identity of countries. For many post-colonial countries, health systems development is an important ingredient in nation-building, with the construction of health facilities and the extension of healthcare to rural populations viewed as indicators of progress and modernisation that are sources of national pride. Similarly, the health systems of the United Kingdom, Germany and Canada are often viewed emblematically as a national characteristic.

Perhaps more importantly, health systems play an essential social function in both shaping and reflecting relations amongst groups of people in a society. For example, national health systems reflect the depth and breadth of the social contract between governments and citizens by determining the scope of health-related rights and entitlements afforded to the citizens or residents. They also shape and reflect the relationship between the different socio-economic segments of society by either reinforcing or mitigating the relationship between social inequalities and health inequalities. The degree to which healthcare is affordable, accessible and responsive is also a critical determinant of social mobility.

Furthermore, much research and evidence have demonstrated how health systems play a significant role in defining the lived experience of being poor, socially disadvantaged or infirm. For example, studies from multiple countries have described how sub-standard and abusive treatment from healthcare providers are viewed as core defining features of what it is to be poor (WHO \& World Bank, 2002). Similarly, the chronic anxiety and fear produced by the lack of protection from the costs of illness, injury and disability are a feature of relative poverty in the USA, even for working families (Himmelstein et al., 2005; Whitehead et al., 2001). The International Labour Organization (ILO) defines social health protection as the provision of organised measures to mitigate the distress caused by the reduction of productivity, stoppage or reduction of earnings, or the cost of necessary treatment that can result from ill health (ILO, 2008).

By shaping how medical services are organised, regulated and delivered, health systems also play a key cultural role in society. Indeed, by helping to define the experience and significance of core human experiences such as birth, death and illness, health systems can 
legitimately be viewed as cultural institutions in their own right, interacting with other cultural institutions such as religion and the arts.

Finally, health systems are also economic institutions. Many health systems incorporate markets of various kinds and sizes, as well as market actors who view health systems through a commercial lens. And, as already noted, investments in health systems are also investments in economic development. Not only are they central to the production of healthy and productive workers, they are also a source of employment and can stimulate growth in other industrial sectors.

There is a tendency to gloss over the fact that health systems have multiple identities and roles and instead view them narrowly in mechanistic terms as a structure or instrument designed to deliver health services. However, a fuller understanding of health systems would place greater emphasis on their social, cultural and economic significance and on the fact that they represent a terrain for the contestation of different ideas and interests.

\subsection{Contestation within Health Systems}

Certain competing ideas and interests are particularly important for determining the design, structure, performance and evolution of health systems. These include different ideological positions about the role and responsibility of the state in guaranteeing access and providing healthcare to all, the role and degree of freedom afforded to markets within health systems, and the degree to which individuals are responsible for their own state of health and wellbeing.

Health policy is also affected by competition and contestation between different groups in society over how the costs and benefits of health systems are shared. Indeed, many authors have noted the fundamentally political nature of healthcare reform and policy-making processes that determine the allocation and distribution of resources and the setting of priorities within the health sector (Barker, 1996; Grindle \& Thomas, 1991; Walt \& Gilson, 1994).

Of particular importance are competing views on how health systems should be financed and to what degree financing is regressive or progressive. Determining the extent to which health systems facilitate the sharing of risk and the costs of healthcare across the whole of society is fundamentally a normative process based on views regarding the obligations of higher-income populations to cross-subsidise the 
needs of lower-income groups, and of the young, fit and healthy to help insure against the costs of being old, disabled and unwell.

Evans (1997) identifies conflicts of interest in health systems financing as being grouped around three main axes. He describes the first as being between 'those who pay for services, and those who are paid for them' and centred around the fact that healthcare expenditure is always translated into healthcare incomes (to those who are paid to provide healthcare). As a consequence, there is always a tension between actors who want to contain costs (taxpayers and governments) and those who want to maximise expenditure (health professionals, pharmaceutical companies, etc). In practice, overall expenditure can be grown by increasing public health budgets and/or expanding the inflow of private health finance through direct out-of-pocket payments or private insurance systems.

It is also the case that different provider groups within the health system will compete with each other to capture healthcare expenditure as income for themselves. Thus doctors may promote ideas and policies to safeguard the pre-eminence of medicine within health systems and stave off competition from other types of healthcare provider. Pharmaceutical companies will promote policies and practices that increase the consumption of proprietary medicines; private insurance companies will lobby against social or national health insurance models and against any regulation of insurance markets that would impinge on their ability to generate profits. While these tensions may be the result of legitimate technical disagreements about the most effective and equitable use of resources in a health system, they may also be the result of competing interests.

The second axis of conflict, according to Evans, is between different groups of payers, and it is mainly centred around the choice of method of health financing and how this determines what share of any given level of health spending will be borne by different groups in society. As financing from general taxation generally places a larger burden on people with higher incomes in contrast to private financing, which places a greater burden on those who become (or are at most risk of becoming) ill, Evans argues that it is no surprise that 'higher-income people tend to support private finance, whereas lower-income people do not'.

Evans' third and final axis of conflict is over access to services and the choice between a universal and equitable public sector health system 
and a mixed public-private system. This conflict is between a single system that limits the advantage to people with higher incomes in accessing better care and a mixed system that gives some people the opportunity to "buy their way to the front of the queue and to ensure that any "rationing" is imposed on someone else'.

The three axes of conflict can also reinforce each other. For example, pressure from higher-income groups to ensure that they can access higher-quality care in the private sector can be reinforced by provider groups lobbying against cost containment and restrictions within a universal system as a way of increasing overall health expenditure.

Finally, it is important to note that while the choices between different health financing models and policies can be framed in political and ideological terms, they can also be framed in non-political and technocratic ways. For example, universal systems of healthcare financing and provision can also be promoted as enabling health systems efficiencies through economies of scale and monopsony power. Equally, mixed systems of financing and provision can be advocated as efficiency measures by creating market competition and encouraging greater levels of individual responsibility for health. This point is pertinent because there is a tendency to cast many debates about health policy and healthcare reforms in technocratic terms to obscure or downplay political and ideological differences.

\subsection{Health Systems Frameworks and Typologies}

Given the complexity of health systems, it is not surprising to find that several frameworks have been developed to help study and evaluate them. Many are designed to help describe and understand the various components that contribute to the primary function of delivering health services.

For example, the WHO 'building blocks' framework comprises six building blocks consisting of 'leadership and governance'; four intermediate blocks consisting of 'financing', 'the health workforce', 'medical products, vaccines and technologies', and 'health information systems'; and a final block consisting of 'service delivery'. A framework used by Roemer (1993) similarly conceptualises four functional components that combine to enable service delivery: (1) financing; (2) production of inputs (e.g. personnel, facilities and 
pharmaceuticals); (3) organisation and structure of the health system; and (4) management.

These and other frameworks tend to share a pattern of having a component related to an organising function of some sort (variously labelled as 'governance', 'stewardship', 'management', 'leadership' and 'regulation'), which is then combined with various categories of inputs required to deliver services (e.g. finance and personnel).

However, while such frameworks describe the various components involved in producing health services, they do not provide a useful basis for understanding the social, political and economic dimensions of health systems. For example, the WHO building blocks framework does not allow for the description or assessment of a health system according to the core issues of how health financing, resource allocation and benefits distribution are structured and organised.

Thus it is necessary also to consider frameworks that speak more to these issues, especially political economy frameworks that are focused on describing the determinants of how the costs and benefits of health systems are distributed across society. Such political economy frameworks typically integrate an analysis of how different stakeholders relate to each other and to certain key functions. Many focus on the roles and powers of government and other public institutions in defining the relationship between financing and healthcare delivery, and determining the mandates, opportunities and powers of other actors, and use this as a basis for creating a typology of health systems (Böhm et al., 2013; Field, 1973; Wendt et al., 2009).

One such approach by Rothgang et al. (2005) highlights the core regulatory function of structuring the relationships between financing agencies, healthcare providers and potential beneficiaries and classifies health systems according to who is involved in carrying out this function. They also break down regulation into six objects: (1) determining which parts of the population are included in the public and/or private system (coverage); (2) determining the system of financing; (3) determining how providers are remunerated; (4) determining which providers have access to markets and financing agencies; (5) determining how patients access providers; and (5) determining the content and range of services available to patients (Rothgang et al., 2005).

Another political economy framework is one used by Roemer to classify health system policies into four broad types: (1) entrepreneurial and permissive; (2) welfare-oriented; (3) universal and comprehensive; 
and (4) socialist and centrally planned (Roemer, 1991). The Organization for Economic Cooperation and Development has also categorised health systems according to three features: (1) whether the prime funding source consists of payments that are made voluntarily or that are compulsory; (2) whether services are provided by direct ownership (e.g. the ministry of health or social insurance agency provides the services itself), by contractual arrangements (e.g. the ministry of health or social insurance agency contracts providers to deliver services), or simply by private providers (paid by direct out-of-pocket payments); and (3) whether services are paid for prospectively or retrospectively (Böhm et al., 2013).

As a final example of the many ways in which health systems can be described and understood, we draw attention to the selection of a set of five key descriptors suggested by Mills and Ranson (2012):

- The dominant method of financing (e.g. tax, social insurance, private insurance, out-of-pocket payments).

- The underlying political philosophy (e.g. capitalist, socialist).

- The nature of state intervention (e.g. to cover the whole population or only the poor).

- The level of gross national product (e.g. low, middle, high).

- Historical or cultural attributes (e.g. industrialised, non-industrialised, transitional).

\subsection{The Open and Contextual Nature of Health Systems}

As noted earlier, health systems are social institutions that can help inform wider social and political norms and attitudes such as those that relate to the relationship between the state and inhabitants or between different segments of society. However, health systems are also clearly affected and influenced by social, economic and political forces that are highly contextual.

For example, the end of the Second World War in western Europe and the end of direct colonialism in Africa and Asia were political events that helped produce an environment that sustained strong government commitment to the idea of universal public sector health systems. Conversely, the collapse of the Soviet Union and the shift from a socialist to a capitalist political system precipitated the collapse of the universal health system in many parts of the former Soviet Union. 
Other examples of political systems influencing the design of health systems include the racist and fragmented structures of the apartheid health system in South Africa and the communitarian health system of Cuba.

We can also see how ideologies and value systems such as democracy and human rights can shape the design of health systems. For example, many countries now have constitutions in which governments have duties to ensure the progressive realisation of human rights, including access to essential healthcare. At the same time, health systems are also affected by political and economic changes. For example, economic recession and a debt crisis in Africa, Asia and Latin America in the 1980s and 1990s led to structural adjustment programmes that produced a set of health sector reforms that included public sector budget cuts and a shifting of the burden of healthcare costs onto households through the use of user fees and private healthcare.

Similarly, neoliberal policies and globalisation have combined to produce a set of political dynamics that have had profound influence on the design and evolution of health systems. These include an increase in cross-border flows of finance, goods and services affecting the health sector and the general adoption of policies aimed at deregulating the health sector, outsourcing healthcare services liberalisation and promoting privatisation. Some of these changes have also been enhanced by technological developments that have facilitated the faster and freer flow of finance and information across national borders.

Any understanding of the evolution, design and performance of a health system must therefore include an analysis of its social, economic and political context. It is also important to examine the wider context when considering policies to develop, strengthen or reform health systems for the future. While health systems are subjected to a variety of external forces and changing circumstances, ideally they should be capable of anticipating new external forces and changes to the context in which they operate. For example, developments in medicine that are driven by technological advances in artificial intelligence, gene therapy, robotics, nanotechnology, cybernetics and telecommunications are likely to create a variety of demands on health systems and healthcare services that can be accommodated either in a planned or an unplanned manner. Similarly, we can anticipate that global warming and climate change, coupled with the likely accompaniment of mass population displacements, will exert pressure on 
healthcare systems in different parts of the world. Other transitions that are currently underway include demographic and nutrition transitions that will probably increase the number of people living in old age and with chronic diseases.

\subsection{Malaysia as a Case Study}

This book tells the story of the evolution of Malaysia's health system. The story is worth telling because it is a health system that has been successful, to a large extent, in having achieved universal health coverage and significant progress in health outcomes of the population. At the same time, Malaysia, like other countries, continues to face a number of complex challenges, including emerging infectious and non-communicable diseases. In telling the story of Malaysia's health system, this book also critically examines the robustness of the foundations of the health system to meet these challenges.

To do so, we have applied a systems-thinking analytical approach. Systems thinking has emerged as an area of health systems research and analysis designed specifically to help decipher the complexity of health systems and inform the design and evaluation of health systemsstrengthening interventions (De Savigny \& Adam, 2009). The practice of systems thinking has developed as we recognise the complexity and interconnectedness of our problems. As a solution to a complex problem and a tool that cuts across disciplines, systems thinking is itself complex. Thus it evades a simple, concise definition and has been subject to multiple definitions and redefinitions (Arnold \& Wade, 2015). For our purposes, we describe systems thinking as an approach to recognising that events take place in the context of a system and that systems are composed of elements that are interconnected through feedback loops such that solely following a linear analysis of cause and effect will fail to capture important understandings of the system. By studying the system as a whole, and not just the sum of its parts, the practice of systems thinking leads to better understanding and better decision-making. We detail our approach and methodology in Chapter 2.

The application of systems thinking and theory to the health sector has received growing attention in recent years. This has resulted in part from frustrations over the limited success of interventions for strengthening and improving the performance of health systems. It also comes from a growing appreciation of the fact that health systems are 
complex adaptive systems in which interactions between system components are typically non-linear, not easily controlled, unpredictable and characterised by unintended effects, paradoxical behaviour and multiple feedback loops (De Savigny \& Adam, 2009; Dorner, 1997; Tan et al., 2005; Rickles et al., 2007; Paina \& Peters, 2011). As such, the WHO has encouraged systems thinking as a way of enabling researchers, funders and policy-makers to improve their understanding of what works in health systems strengthening and 'catalyse conceptual thinking regarding health systems, system-level interventions, and evaluations of health system strengthening' (De Savigny \& Adam, 2009).

We aim to demonstrate several ways that systems methodologies can contribute to health system strengthening. Systems thinking is a useful means of exploring problem spaces and boundaries, including linkages within and beyond the health system. Health system problems and interventions do not respect categorical or highly structured categories, such as health system building blocks, nor disciplinary and organisational boundaries, such as the various departments in a health ministry. In tracing the feedback loops to describe a system surrounding a problem, systems thinking helps us re-evaluate our assumptions about what is and isn't relevant. It helps us think about contexts as dynamic and interactive rather than as a static background that remains unchanged in an intervention. It also draws our attention to the deep connections between societies and health systems and the ways they shape one another.

The systems' interactions in health systems are inherently complex. The visual representation of causal pathways in systems thinking can help us understand and communicate such complexity. Likewise, the emphasis on feedback loops as system drivers focuses our attention on the self-corrective and adaptive capacities of systems and away from deceptively simple narratives of linear cause and effect. By providing a framework and language for thinking and speaking about complexity, systems thinking increases our capacity to engage meaningfully with it.

Finally, by redefining problem spaces and facilitating conversation about complex systems, systems thinking fosters interdisciplinarity and co-production for health systems strengthening. The systems analysis and visualisation in this book came out of extensive research, consultation and dialogue with a wide range of stakeholders that fed into an iterative process of sense-making between subject matter experts and 
systems thinkers. The process of co-production not only makes the knowledge generated more holistic and robust but also increases the likelihood of use and acceptance due to buy-in and the creation of an output that is understandable to all partners involved.

The book is divided into three sections. Section I consists of two introductory chapters. The first unpacks the multiple dimensions of a 'health system' and discusses the different ways in which a health system is complex. It describes health systems from a number of dimensions, including the technocratic, social, cultural, political, economic and normative. In doing so, it describes a variety of health systems frameworks and typologies. It also discusses the importance of contextualising any description and analysis of any health system and understanding how health systems thinking and policy have evolved over time internationally. The second introductory chapter presents the aspects of systems thinking and systems theory that are the focus of this book. It describes the growing interest in applying systems thinking to the study of health systems, the challenges faced, and the dual approach of case studies and whole-systems analysis taken herein.

In Section II, we present the story of the development of the Malaysian health system, its changes over time and the challenges it faces today and looking forward. It begins with a historical overview of the health system in Chapter 3, followed by separate chapters on each of the WHO health systems building blocks. The service delivery building block is divided into four sections: primary care, secondary and tertiary care, disease control, and environmental health. In each chapter, systems thinking is applied to examine the development of each building block and their linkages with each other as well as the drivers, enablers and obstacles outside the health system. The division of thematic areas into building blocks is purely instrumental.

Finally, Section III presents some generic lessons that can be used to guide future action and future change in both Malaysia and elsewhere. Chapter 13 summarises and discusses a number of issues and observations that cut across the various chapters in Section II and considers the challenges facing the Malaysian health system today and in the future. The chapter also highlights lessons that may be applicable to other countries in Asia and Africa. Chapter 14 presents lessons on the functioning and behaviour of health systems through reflections on the development of the case studies and an analysis of the key feedback processes that shape health systems. 


\section{References}

Arnold, R. D. and Wade, J. P. (2015). A definition of systems thinking: a systems approach - ScienceDirect. Procedia Computer Science, 44, 669-78.

Barker, C. (1996). The Health Care Policy Process. Thousand Oaks, CA: Sage.

Böhm, K., Schmid, A., Götze, R., Landwehr, C. and Rothgang, H. (2013). Five types of OECD healthcare systems: empirical results of a deductive classification. Health Policy, 113, 258-69.

De Savigny, D. and Adam, T. (2009). Systems Thinking for Health Systems Strengthening. Geneva: World Health Organization.

Dorner, D. (1997). The Logic of Failure: Recognizing and Avoiding Error in Complex Situations. New York: Basic Books.

Evans, R. G. (1997). Health care reform: who's selling the market, and why? Journal of Public Health, 19, 45-9.

Field, M. G. (1973). The concept of the 'health system' at the macrosociological level. Social Science \& Medicine, 7, 763-85.

Gilson, L. (2003). Trust and the development of health care as a social institution. Social Science \& Medicine, 56, 1453-68.

Grindle, M. S. and Thomas, J. W. (1991). Public Choices and Policy Change: The Political Economy of Reform in Developing Countries. Baltimore, MD: Johns Hopkins University Press.

Himmelstein, D. U., Warren, E., Thorne, D. and Woolhandler, S. (2005). Illness and injury as contributors to bankruptcy. Health Affairs, 24, W563.

International Labour Organization. (2008). Social Health Protection: An ILO Strategy towards Universal Access to Health Care. Geneva: International Labour Organization.

Mills, A. J. and Ranson, M. K. (2012). The design of health systems. In M. H. Merson, ed., Global Health: Diseases, Programs, Systems, and Policies. Burlington, MA: Jones \& Bartlett Learning, pp. 615-48.

Paina, L. and Peters, D. H. (2011). Understanding pathways for scaling up health services through the lens of complex adaptive systems. Health Policy and Planning, 27, 365-73.

Rickles, D., Hawe, P. and Shiell, A. (2007). A simple guide to chaos and complexity. Journal of Epidemiology \& Community Health, 61, 933-7.

Roemer, M. I. (1991). National Health Systems of the World. Vol. I. Oxford: Oxford University Press.

Roemer, M. I. (1993). National Health Systems of the World. Vol. II. Oxford: Oxford University Press. 
Rothgang, H., Cacace, M., Grimmeisen, S. and Wendt, C. (2005). The changing role of the state in healthcare systems. European Review, 13, 187-212.

Sachs, J. D. (2001). Macroeconomics and Health: Investing in Health for Economic Development. Geneva: World Health Organization.

Tan, J., Wen, H. J. and Awad, N. (2005). Health care and services delivery systems as complex adaptive systems. Communications of the ACM, 48, 36-44.

Walt, G. and Gilson, L. (1994). Reforming the health sector in developing countries: the central role of policy analysis. Health Policy and Planning, 9, 353-70.

Wendt, C., Frisina, L. and Rothgang, H. (2009). Healthcare system types: a conceptual framework for comparison. Social Policy \& Administration, 43, 70-90.

Whitehead, M., Dahlgren, G. and Evans, T. (2001). Equity and health sector reforms: can low-income countries escape the medical poverty trap? The Lancet, 358, 833-6.

World Health Organization and World Bank. (2002). Dying for Change: Poor People's Experience of Health and Ill-Health. Washington, DC: World Bank. 